Ann. Biol. anim. Bioch. Biophys., I975, 15 (4), 765-769.

\title{
ÉVOLUTION DU DÉCIDUOME EXPÉRIMENTAL ET DU BLASTOCYSTE EN LÉTHARGIE CHEZ LA RATTE
}

\author{
I. 一 LE DÉCIDUOME EXPÉRIMENTAL \\ CHEZ LA RATTEE EN GROSSESSE UNILATÉRALE
}

H. DUPONT, C. ESNAULT*, Anne-Josette DULUC et G. MAYER avec la collaboration technique de Liliane Despuyoos (I. N. S. F. R. M.) et de J. C. Nicolle (I. N. R. A.).

Laboratoire d'Histologie et Embryologie,

Université de Bordeaux $I I$,

146, rue Léo-Saignat,

33076 Bordeaux.

* Station de Physiologie de la Reproduction,

Centre de Recherches de Tours, I. N.R.A.,

Nouzilly, 37380 Monnaie

\section{RÉSUMÉ}

L'évolution chronologique des différents types nucléaires du déciduome expérimental chez la Ratte est étudiéc par des techniques de cytogénétique et de cytophotométrie.

Cette étude a montré que la genèse des cellules binucléées précède celle des cellules mononucléées de la classe polyploïde immédiatement supérieure. Ce tissu décidual évolue de $2 n$ à $32 n$ en 3 jours puis đégénère.

On sait depuis les travaux de LOEB en rgo7 [Io] qu'un traumatisme utérin pendant la phase progestative provoque dans l'endomètre lésé une réaction tissulaire aboutissant à la formation d'une petite tumeur, le déciduome (CoURRIER [4]). Chez la Ratte cette réaction déciduale traumatique, semblable à celle provoquée physiologiquement par l'implantation de l'œuf, apparaît d'abord dans la région antimésométrale pour s'étendre progressivement vers la région mésométrale [8], [I8]. L'apparition et l'évolution de ce tissu décidual sont soumises à un strict conditionnement hormonal caractérisé par un équilibre précis entre progestérone et ostrogène $[5]$. 
Depuis la description classique de Krehbiel [8] relative à l'étude cytologique de la réaction déciduale chez le Rat, toutes les publications mentionnent le polymorphisme nucléaire de ce tissu [5]. VELARDO en I953 [I7] suggère d'après la taille des noyaux un certain degré de polyplö̈die. Ein I955, SAchs et SHELESAYak [I5] utilisant la technique du "Squash » nucléaire afin d'obtenir une bonne dispersion chromosomique [I4], évaluent le degré de polyploïde de ce tissu en fonction du comptage chromosomique et de la taille des noyaux. Ces différents auteurs [5], [8], [I5], [I7], signalent également la présence de cellules binucléées.

Nos recherches ont pour but d'explorer l'évolution des cellules déciduales au cours d'une grossesse unilatérale, c'est-à-dire en présence d'un équilibre hormonal physiologique afin de déterminer si ce tissu est le résultat d'une prolifération anarchique ou s'il est régulé selon certains mécanismes définis.

\section{MATÉRIEL ET TECHNIQUES}

\section{I. - Technique de décidualisation}

Les déciduomes expérimentaux ont été obtenus de la manière suivante. Des Rattes vierges de souche Wistar pesant de I 50 à $180 \mathrm{~g}$ sont mises au mâle fécond. La présence d'un bouchon vaginal date le r er jour de la grossesse. Ce même jour à ıo heures on sectionne la trompe gauche au niveau de la jonction tubo-utérine, a fin d'obtenir une corne utérine stérile. Les ovoimplantations qui ont lieu dans la corne droite fertile servent de témoin de l'équilibre hormonal gravidique. Le jour 5 ( $\mathrm{IO} \mathrm{h}$ ) on injecte dans la corne utérine gauche stérile $0,05 \mathrm{ml}$ d'huile d'arachide. Les animaux sont sacrifiés du $6^{\mathrm{e}}$ au $1^{\mathrm{e}}$ jour de la grossesse, c'est-à-dire de 24 à $\mathbf{2} 20$ h après le traumatisme déciduogène.

\section{2. - Technique de cytogénétique}

A fin de réaliser le caryotype des cellules déciduales les mitoses sont bloquées en métaphase par l'administration intrapéritonéale de colchicine à la dose de I $\mu g / g$ de poids [I6] $4 \mathrm{~h}$ avant le sacrifice. Les déciduomes sont prélevés et dissociés au microbroyeur dans une solution physiologique. Après centrifugation à $500 \mathrm{tr} / \mathrm{mn}$ pendant $5 \mathrm{mn}$ le surnageant est rejeté et le culot cellulaire est fixé directement ou laissé pendant $30 \mathrm{mn}$ en suspension dans une solution hypotonique constituée de sérum de veau au $\mathrm{x} / 5$ additionné d'hyaluronidase à $37^{\circ} \mathrm{C}(0,5 \mathrm{ml}$ à 50 UI pour Io ml de solution hypotonique). Après une nouvelle centrifugation, le culot cellulaire est fixé dans du Carnoy pendant $\mathrm{I}$ h. Puis on fait tomber 2 gouttes de la suspension cellulaire sur une lame froide et propre. Une fois séchée la préparation est colorée au Giemsa ou au Bleu de Toluidine tamponné par une solution à $\mathrm{pH}$ 4,66 pour la mise en évidence des corpuscules de Barr sur préparation fixée au Carnoy sans choc hypotonique.

Cette technique a l'avantage d'un bon étalement nucléaire qui rend plus aisée l'observation et la numération des différentes classes nucléaires. Elle permet de déterminer le degré de polyploïde des noyaux :

- d'après l'établissement du caryotype pour les cellules en mitose ;

- pour les cellules en interphase, d'après la présence d'un ou plusieurs corpuscules de Barr [r], [ 3 3]; le diamètre et le nombre de nucléoles [20].

\section{3. - Technique cytophotométrique}

La coloration de Feulgen s'effectue dans des conditions rigoureusement standardisées. Une lame témoin sert de contrôle. Le temps d'hydrolyse nécessaire à la fixation optimale du colorant Feulgen sur les radicaux aldéhydes libérés est déterminé en réalisant une hydrolyse par $\mathrm{HCl}$ normal à $60^{\circ} \mathrm{C}$ durant $6,8,10,12$, I 4 et $16 \mathrm{~mm}$. 30 mesures sont effectuées par microspectrophotométrie pour chaque temps d'hydrolyse, le maximum de colorant Feulgen fixé est défini par un temps d'hydrolyse de $10 \mathrm{mn}$. Les lames servant à. l'expérinentation sont ensuite préparées dans des conditions rigoureusement semblables après une hydrolyse $\mathrm{HCl} \mathrm{N}$ à $60^{\circ} \mathrm{C}$ de Io mn. Les mesures 
d'ADN sont réalisées en lumière monochromatique à 560 mu par " l'Lniversalmikrospektrophotometer UMSPI Zeiss it platine rapide donnant automatiquement en unités arbitraires par affichage digital l'extinction totale du noyau soit le produit de la densité optique par le coefficient d'intégration de l'appareil. Cette technique par balayage est plus précise que la méthode du "bouchon "lorsque la distribution du matérict absorbant est hétérogène [3].

\section{RÉSULTATS}

\section{- I. - Variétés cellulaires}

Nous avons effectué une étude statistique sur l'évolution chromosomique et cytologique du déciduome expérimental pendant les 5 premiers jours [6].

Nous avons défini 5 classes nucléaires:

- les cellules mononucléées diploïdes à chromatine dense ;

- les cellules mononucléées diploïdes à chromatine finement dispersée ;

- les cellules binucléées diploïdes ;

- les cellules mononucléées polyploïdes $(4 n$ et +$)$;

- les cellules binucléées polyploïdes $(4 n$ et +$)$.

\section{2. - Evolution dans le temps des différents types cellulaires}

— vingt-quatre heures après le traumatisme utérin, la majorité de la population cellulaire est représentée par des cellules mononucléées diploïdes à chromatine dense. L,es cellules mononucléées diploïdes à chromatine finement dispersée apparaissent. Quelques cellules binucléées se forment. Les autres classes nucléaires ne sont pas encore représentées.

- De 48 à 96 heures, on observe simultanément une diminution des cellules diploïdes et une augmentation des cellules binucléées diploïdes, mononucléées polyploïdes et binucléées polyploïdes.

- Au $4^{\mathrm{e}}$ jour le nombre de cellules binucléées diploïdes diminue alors qu'augmente la population des cellules mononucléées polyploïdes.

Puis ce tissu dégénère. Il subsiste la partie profonde du stroma constituée par des cellules mononucléées diploïdes.

Les mesures d'ADN-Feulgen par cytophotométrie [7] ont précisé ces résultats.

- vingt-quatre heures après le traumatisme, le chorion endométral est peu différencié. Les noyaux ont une valeur ADN-Feulgen de 12,66 représentant un génome diploïde, servant de référence aux autres classes nucléaires.

- A 48 heures, les valeurs individuelles des cellules mononucléées sont groupées par palier à $2 n, 4 n, 8 n$. Peu de cellules nononucléées parviennent au stade $\mathbf{r} 6 n$. Les cellules binucléées de $4 n$ à I $6 n$ ont une évolution semblable aux mononucléées.

- A 72 heures, ce tissu présente une grande variété nucléaire. Les noyaux en phase $\mathrm{S}$ sont nombreux. Certaines cellules mononucléées possèdent un génome $32 n$. La polyploïdie est plus importante qu'au jour précédent. Une nouvelle classe I $6 n$-32n apparaît.

- A 96 heures et 120 heures bien que les noyaux aient une chromatine d'aspect laqué en voie de lyse, les valeurs ADN-Feulgen représentant l'évolution maximale sont extrêmement élevées. 
Les cellules binucléées inférieures à $4 n$ montrent une perte en ADN-Feulgen importante. Les nuages de points des cellules mono et binucléées tendent à se superposer illustrant un état de dégénérescence plus avancé des cellules binucléées.

Dans la région mésométrale, la plus vascularisée de l'endomètre, les cellules déciduales évoluent très différemment sous l'effet du traumatisme utérin. Les cellules stromales ne dépassent pas $4 n$.

- Les cellules mono et binuclées mésométrales au jour 7, 8 et 9 synthétisent activement leur ADN. Leurs noyaux sont en $\mathrm{G}_{1}$, S et $\mathrm{G}_{2}$. On n'observe pas de différence dans le rythme de synthèse.

- Au jour Io, on note l'absence de synthèse d'ADN dans les cellules binucléées qui sont fréquemment le siège de scissiparité nucléaire donnant 2 cellules filles mononucléées tétraploïdes.

Par contre les cellules mononucléées sont toujours en période d'activité synthétique.

- Le II jour, les cellules binucléées sont en $\mathrm{G}_{1}$ et les mononucléées groupées en $G_{1}$ et $G_{2}$ avec quelques rares valeurs de synthèse.

\section{DISCUSSION}

Le mode de formation des noyaux cellulaires polyploïdes dans le tissu décidual rappelle celui observé dans le foie des mammifères [II]. Les cellules binucléées représenteraient un stade transitoire vers la polyploïdie. L'apparition des cellules binucléées précède celle des cellules mononucléées de la classe polyploïde immédiatement supérieure. Selon BEams [2], Wilson [I9], NADAL et ZajDELA [II], les cellules binucléées du tissu hépatique se diviseraient par un seul fuseau pour donner 2 cellules filles mononucléées de la classe polyploïde supérieure. Dans le tissu décidual les noyaux des cellules binucléées entrent simultanément en division. A la fin de la prophase, les 2 équipements chromosomiques sont encore identifiables et délimitent les sites des 2 anciens noyaux. Puis au cours de la métaphase, les 2 lots de chromosomes s'unissent. Des études ultrastructurales en cours semblent confirmer 1'hypothèse de NADAL, d'un seul fuseau pour les cellules binucléées en mitose. Ces mitoses fusoriales mono et binucléées sont suivies d'endomitoses mono et binucléées.

Ce tissu possède un pouvoir de synthèse exceptionnel puisqu'il duplique son matériel nucléaire de $2 n$ à $32 n$ e: 3 jours. Nos résultats sont en accord avec ceux de LEROY [9]. Dans la région mésonétrale ce tissu présente les premiers stades d'évolution vers la polyploïdie par l'intermédiaire de cellules binucléées sans pouvoir accéder à un degré de ploïdie supérieur à $4 n$.

Colloque D. G. R. S. T., Biologie de la Procréation, Paris, 7-8 mars 1975.

\section{REMERCIEMENTS}

Ce travail a été réalisé grâce à l'aide de la D. G. R. S. T., contrat no 7r-7-3247. 


\section{SUMMARY}

\section{EVOLUTION OF EXPERIMENTAL DECIDUA}

AND DORMANT BLASTOCYST IN THE FEMALF RAT.

\section{I. - EXPERIMENTAL, DECIDUA IN UNILATERAI, GESTATION}

The chronological evolution of the different types of nuclei in decidual tissue was studied with cytogenetic and cytophotometric techniques in the Rat. This studies had shown that genesis of binucleated cells preceded genesis of mononucleated cells belonging to superior polyploid type. This decidual tissue evolves from $2 n$ to $32 n$ within three days and then degenerates.

\section{RÉFÉRENCES BIBLIOGRAPHIQUES}

[I] Barr M. L., Bertram E. G., I949. A morphological distinction between neurones of the male and female and the behavior of the nucleolar satellite during accelerated nucleoprotein synthesis. Nature, 163, 676-677.

[2] Beams H. W., King R. L., I942. The origin of binucleate and large mononucleate cells in the liver of the rat. Anat. rec., 83, $28 \mathrm{I}$.

[3] Caspersson T. O., Lomakka M., ig62. Scanning microscopy techniques for high resolution quantitative cytochemistry. Ann. New-York Acad. Sc., 97, 449-462.

[4] Courrier R., I945. Endocrinologie de la gestation. Masson Edit., Paris.

[5] De Feo V. J., 1967. Cellular biology of the uterus. Wynn R. M., North Holland Publ. Comp., Amsterdan, $192-290$

[6] Dupont H., Duluc A. J., Mayer G., I97I. Évolution cytologique et genèse de la polyploïdie dans le déciduome expérimental chez la Ratte en gestation unilatérale. C. R. Acad. Sc. Paris, 272, D, 2360-2363.

[7] Dupont H., Esnault C., Duluc A. J., Mayer G., 1974. Évolution de la polyploïdie dans le déciduome expérimental chez la Ratte en grossesse unilatérale : étude cytophotométrique. $C$. $R$. Acad. Sc. Paris, 279, D, 50I-504.

[8] Krenbiel R. H., i937. Cytological studies of the decidual reaction in the rat during early pregnancy and in the production of deciduomata. Physiol. Zool., 10, 212-234.

[9] Leroy F., I974. Étude fonctionnelle de l'acide désoxyribonucléique dans l'endomètre du rongeur. Analyse morphologique semi-quantitative. Collection " Medico-Monographies d'Agrégés ", Arscia S. A., Bruxelles, p. 98-I04.

[Io] Loeb L., I907. Uber die experimentalle Erzeugung von knoten von deciduagewebe in dem uterus des meerschweinchens nach stattgefundener Copulation. $Z$ bl. Allg. Path., 18, 563-565.

[II] Nadal C., Zajdela F., Ig66. Polyploidie somatique dans le foie de Rat. Le rôle des cellules binucléées dans la genèse des cellules polyploïdes. Exp. Cell. Res., 42, 99-I 6.

[12] Nadal C., I97o. Polyploïdie hépatique du Rat. Mode de formation des cellules binuclées. $J$. Microscopie, 9, 61-618.

[13] Oнno S., r969. Chromosomes sexuels et gènes liés au sexe. Gauthier-Villars, Paris, p. 87-9r.

[I4] Sachs L., I953. Simple methods for mammalian chromosomes. Stain Tech., 28, x69-I72.

[I5] Sachs L., Shelesnyak M. C., I955. The development and suppression of polyploidy in the developing and suppressed deciduoma in the rat. $J$. Endocr., 12, I46-I5I.

[I6] TARkowski A. R,, Ig66. An air-drying method for chromosomal preparations from mouse eggs. Cytogenetics, 5, 394-400.

[i7] Velardo J. T., Dawson A. B., Olsen A. G., Hisaw F. L,, I953. Sequence of histological changes in the uterus and vagina of the rat during prolongation of pseudo-pregnancy associated with the presence of deciduomata. Amer. J. Anat., 93, 273-305.

[18] Velardo J. T., 1958. Endocrinology of reproduction. Oxford Univ. Press, Londres et New York.

[I9] Wilson J. W., Leduc E. H., I948. The occurrence and formation of binucleate and mononucleate cells and polyploid nuclei in the mouse liver. Am. J. Anat., 82, 353-39r.

[20] ZajDELA F., LANGLors M., I955. Constance des rapports nucléo-nucléolaires dans le foie de souris au cours de la croissance et du jeûne. C. R. Soc. Biol. 149, 305-307. 